\title{
Automedicación odontológica en pacientes que acuden al servicio público y privado, Azogues-2018
}

\author{
Self-medication in dental patients who use public and private services, \\ Azogues-2018 \\ Automedicação odontológica em pacientes que vêm ao serviço \\ público e privado, Azogues-2018
}

\author{
Gabriela Araujo Crespo ${ }^{1}$ \\ Cristina Crespo Crespo ${ }^{2}$ \\ Cristina Domínguez Crespo ${ }^{3}$ \\ Fernando Vallejo Aguirre ${ }^{4}$
}

Recibido: 15 de agosto de 2018

Aprobado: 4 de diciembre de 2018

Publicado: 1 de julio de 2019

Cómo citar este artículo: Araujo Crespo G, Crespo Crespo C, Domínguez C, Vallejo-Aguirre F.

Automedicación odontológica en pacientes que acuden al servicio público y privado, Azogues-2018.

Rev Nac Odontol. 2019; 15(29):1-17 doi: https://doi.org/10.16925/2357-4607.2019.02.02

Artículo de investigación. https://doi.org/10.16925/2357-4607.2019.02.02

1 Universidad Católica de Cuenca sede Azogues, Ecuador

ORCID: https://orcid.org./0000-0001-7120-8616

Gabriela Araujo Crespo, Avenida 16 de Abril, y Ernesto Che Guevara, Azogues, Ecuador

Correo electrónico: gabyfra8@gmail.com

2 Universidad Católica de Cuenca sede Azogues, Ecuador ORCID: https://orcid.org/0000-0002-2398-217X

3 Universidad Católica de Cuenca sede Azogues, Ecuador ORCID: https://orcid.org/0000-0002-0761-9493

4 Universidad Católica de Cuenca sede Azogues, Ecuador ORCID: https://orcid.org/0000-0002-6869-723 


\section{Resumen}

Introducción: la automedicación es un problema de salud pública, pudiendo generar reacciones adversas, resistencia bacteriana; provocando en el paciente un riesgo mayor, que puede ser evitable, costoso e ineficaz. En odontología el dolor dental es uno de los principales motivos de automedicación, pues la gran mayoría de personas lo manejan a través de la ingesta de fármacos sin prescripción médica, por ello, el objetivo de este estudio fue identificar la prevalencia en pacientes de establecimientos públicos y privados en el cantón Azogues, Ecuador.

Materiales y métodos: estudio observacional, descriptivo y transversal, la muestra estuvo conformada por 201 pacientes de ambos sexos, de 17 a 60 años; se aplicó un cuestionario validado. Resultados: La prevalencia general fue de $75 \%$, en la entidad privada más prevalente en sexo masculino $60 \%$, y en la pública femenino $49 \%$; los AINES fueron los fármacos más usados en ambos sexos; el grupo que más se automedicó fue de 17 a 25 años, prevaleciendo la automedicación con AINES 33 \%; la presentación más usada según edad y sexo fueron las tabletas sin relación estadísticamente significativa, y la patología dental asociada a automedicación fue dolor dental.

Resultados y conclusiones: la automedicación odontológica fue practicada por las dos terceras partes de la muestra, hubo mayor prevalencia en sexo femenino y en los grupos de 17 a 25 años, el fármaco más usado fue AINES y la forma más usual las tabletas. La mayoría de los encuestados están conscientes del daño que podría ocasionar la automedicación en la salud.

Palabras clave: automedicación odontológica, AINES, fármaco.

\section{Abstract}

Introduction: Self-medication is a public health problem as it can generate adverse reactions and bacterial resistance, causing greater risk to the patient. It is expensive, ineffective and can be avoidable. In dentistry, dental pain is one of the main reasons for self-medication, since the vast majority of people manage it through the intake of drugs without medical prescription. Therefore, the objective of this study was to identify its prevalence in patients of public and private establishments in the canton of Azogues, Ecuador.

Materials and methods: An observational, descriptive and cross-sectional study was performed. The sample consisted of 201 patients of both sexes, aged 17 to 60 years. A validated questionnaire was applied.

Results: The general prevalence was $75 \%$, with the highest prevalence among males in the private sector $60 \%$, and in females in the public sector $49 \%$. NSAIDs were the most commonly used drugs by both sexes; the group that self-medicated most was from 17 to 25 years, with $33 \%$ self-medicating with NSAIDs $33 \%$. The most popular way for taking the drugs, according to age and sex, was in tablet form (without a statistically significant relationship), and the dental pathology associated with self-medication was dental pain.

Results and conclusions: Dental self-medication was practiced by two thirds of the sample, there was a higher prevalence in females and in the age group of 17 to 25 years, the most used drug was NSAIDs and the most common form was tablets. Most respondents are aware of the damage that self-medication can cause to health.

Keywords: dental self-medication, NSAIDs, medication.

\section{Resumo}

Introdução: A automedicação é um problema de saúde pública, pois pode gerar reações adversas e resistência bacteriana, causando maior risco ao paciente. É caro, ineficaz e pode ser evitável. Na odontologia, a dor dentária é um dos principais motivos da automedicação, uma vez que a grande maioria das pessoas a administra através da ingestão de medicamentos sem receita médica. Portanto, o objetivo deste estudo foi identificar sua prevalência em pacientes de estabelecimentos públicos e privados no cantão de Azogues, Equador. 
Materiais e métodos: Foi realizado um estudo observacional, descritivo e transversal. A amostra foi constituída por 201 pacientes de ambos os sexos, com idades entre 17 e 60 anos. Um questionário validado foi aplicado.

Resultados: A prevalência geral foi de $75 \%$, sendo a mais alta entre os homens do setor privado $60 \%$ e entre as mulheres do setor público $49 \%$. Os AINEs foram os medicamentos mais usados por ambos os sexos; o grupo que mais se automedicou foi de 17 a 25 anos, com 33\% se automedicando com AINEs 33\%. A maneira mais popular de tomar os medicamentos, de acordo com a idade e o sexo, foi na forma de comprimidos (sem uma relação estatisticamente significativa), e a patologia dentária associada à automedicação foi a dor dentária.

Resultados e conclusões: A automedicação odontológica foi praticada por dois terços da amostra, houve maior prevalência no sexo feminino e, na faixa etária de 17 a 25 anos, o medicamento mais utilizado foi o uso de AINEs e a forma mais comum foi a de comprimidos. A maioria dos entrevistados está ciente dos danos que a automedicação pode causar à saúde.

Palavras-chave: automedicação odontológica, AINEs, medicação.

\section{Introducción}

En la actualidad uno de los principales problemas de salud que aqueja a la sociedad es el dolor, de tal manera quienes lo padecen buscan encontrar alivio mediante el consumo de fármacos, ya sean estos analgésicos, o antiinflamatorios no esteroides (AINES), los cuales son adquiridos en farmacias o tiendas, muchas veces sin prescripción médica $(1,2)$.

En el área de la salud, especialmente médica y odontológica, se posiciona a la automedicación como un fenómeno ampliamente discutido, ya que constituye una práctica común dentro de los autocuidados que el ser humano realiza por su salud con sus respectivas connotaciones positivas y negativas. El origen de la automedicación puede ser por varias razones, su prevalencia está influenciada por diversos factores y, según algunos estudios, se determina que las condiciones socioeconómicas, culturales del individuo, hábitos; así como también la publicidad farmacéutica son otras de las causas que contribuyen a esta cuestionada práctica (3). A ello se puede sumar lo referido en ciertos estudios en donde los antibióticos y especialmente los que contienen dos o más principios activos son de uso preferencial, utilizados especialmente en molestias agudas como los abscesos periapicales, considerándose por tanto que el campo de la profesión puede ser un eventual factor de riesgo para una medicación irresponsable e indiscriminada en razón de la urgencia por solucionar una molestia aguda, y la facilidad con la que en un tiempo a se podía conseguir fácilmente el expendio de dichos antibióticos (4).

De acuerdo con la Organización Mundial de la Salud (OMS), la automedicación se define como la selección y el uso de medicamentos por parte del usuario para el tratamiento de enfermedades o síntomas reconocibles por él mismo; otros autores 
la relacionan con el consumo inapropiado de fármacos para combatir patologías autodiagnosticadas; ante esto se afirma que tanto la obtención de recetas como las prácticas de consulta no médicas son propias de países en vías de desarrollo $(1,5,6)$

La automedicación desde el año de 1980 ha sido considerada por la OMS como uno de los aspectos más importantes dentro de la Salud Pública, cabe recalcar que este fenómeno universal tiene una frecuencia variada de hasta un 68 \% en Europa, $31 \%$ en la India y 59 \% en Nepal. En los países occidentales este fenómeno llamado automedicación es atribuido a un sistema médico pluralista que se enfoca en la medicina tradicional $(6,7)$.

Ciertos datos obtenidos en países sudamericanos como Perú determinan que la automedicación odontológica es un fenómeno, que se produce de manera importante, no solo en razón de la venta libre que existe, sino también fruto de la prescripción médica, aspectos que conducen a un uso irracional de los medicamentos, ello especialmente en adultos y adultosjóvenes en un rango de 18-50 años de edad, reportándose que, según estos estudios, debido a la principal dolencia odontológica que constituye el dolor dental los medicamentos más autoadministrados son los analgésicos y los AINES, y en la mayoría de los casos por recomendación farmacéutica, o por la influencia de los medios de comunicación (8). En Perú, en las ciudades de Cajamarca, Chiclayo, Ica y en Lima se realizaron estudios comparativos acerca de la automedicación odontológica en la consulta pública y privada determinando que más del $69 \%$ de los entrevistados se automedican (9).

En América y Europa se han realizado estudios acerca de la automedicación en poblaciones universitarias del área médica y odontológica en países como Argentina, en donde este hábito es practicado por el $85 \%$ de los universitarios, mientras que en España específicamente en Alicante y Madrid han arrojado cifras de 63 \% y 30,9\% respectivamente (2).

En el Ecuador la automedicación es más común de lo que se estima y las causas son múltiples: publicidad excesiva, necesidad de ahorro en la consulta médica y dependencia. En una encuesta realizada a 20 personas en Quito por el diario El Telégrafo en el 2014, más de la mitad afırmó haber ingerido medicamentos sin prescripción médica. La mayoría reconoce que la automedicación puede ser perjudicial para la salud; en tal virtud se infiere que la automedicación es un problema de Salud Pública en el Ecuador, así como en otros países, y se requieren ejecutar estrategias que informen a la población acerca del gran valor de asistir a los servicios profesionales cuando se necesite de un tratamiento farmacológico (10).

Automedicarse involucra diferentes riesgos potenciales, su consumo desmedido se le enmarca como un problema de salud pública que conlleva ciertos aspectos 
negativos, desde la disminución de la eficacia clínica, hasta sufrir los efectos adversos de los fármacos consumidos, pudiendo también enmascarar una enfermedad subyacente; ello ocurre especialmente en ciertos grupos poblacionales en donde, por la deficiente cobertura de salud pública, optan por una automedicación como solución rápida e inmediata, especialmente cuando están siendo afectados por el dolor dental considerado, según varios estudios, como la primera y principal causa de autoadministración de fármacos. No se debe dejar de considerar que las consecuencias podrían desencadenar desde alergias hasta cuadros graves de insuficiencia renal o hepática; adicionalmente los grupos vulnerables son los más expuestos a sufrir los efectos tóxicos de un fármaco usado de forma inadecuada (8-10).

Es por eso que este trabajo de investigación pretender mejorar o brindar un mejor conocimiento en pacientes de 17 a 60 años de edad que acudieron a las instituciones referidas, pues son espacios de salud de gran afluencia en donde se aprovechó para indagar la problemática planteada, y tener una referencia o un punto de partida.

La información acerca de este tema data de tiempo atrás, pero siempre toma vigencia dada su connotación implícita, y permitirá que los profesionales de la salud, entre ellos al odontólogo, obtengan una cultura de rechazo hacia la automedicación por los riesgos que ella conlleva. En contexto, la investigación estuvo centrada en determinar la frecuencia y el tipo de fármacos más utilizados por parte de los pacientes que acuden tanto a una entidad privada como pública a la hora de sentir molestias odontológicas; ya que al existir escasa información con respecto a este tema que aqueja a la población mundial se quiso realizar una comparación entre dichas entidades y observar entre otros aspectos relevantes si los pacientes tienen conocimiento de los efectos dañinos que tiene esta mala práctica.

Por lo expuesto, el objetivo de este estudio fue describir la automedicación en pacientes que acuden tanto a un servicio público como privado, tomando como referentes a las entidades privadas Clínicas de Especialidades Odontológicas de la Universidad Católica de Cuenca ubicados en Azogues y Cuenca, y al Centro público de Salud No. 1 en Azogues.

\section{Materiales y métodos}

La presente investigación tuvo un enfoque cuantitativo de tipo observacional, diseño descriptivo y de corte transversal. La población de estudio estuvo conformada por todos los pacientes que acudieron a los centros públicos y privados seleccionados para el estudio, esto es pacientes adultos de ambos sexos, en edades comprendidas entre los 17 a 60 años de edad, a quienes se les pidió su expresa 
autorización mediante un consentimiento informado, excluyéndose aquellos que no lo hicieron.Para el cálculo del tamaño muestral se utilizó la herramienta informática medica Fisterra y luego de aplicar los criterios de selección la muestra final quedó constituida por 201 pacientes.

La ficha de recolección de datos estuvo estructurada en dos secciones, la primera que corresponde a datos de filiación, y una segunda en donde se colocaron datos específicos de la automedicación, mediante un cuestionario, que permitió determinar la prevalencia según edad y sexo en la población estudiada, frecuencia de automedicación, edad de la primera automedicación, motivo de automedicación, tipo de medicamento más requerido para la autoadministración, así como también la presentación más utilizada para esta práctica y la persona que lo recomendó. El cuestionario empleado tuvo como referencia el instrumento validado por Conhi et al. (1).

Se solicitaron los permisos respectivos a las entidades involucradas; posteriormente para el cumplimiento de los criterios de selección solo se consideraron aquellos pacientes que firmaron su consentimiento informado. Los datos obtenidos se analizaron mediante el programa Excel 2016 y SPSS v.23, elaborando previamente una base de datos con el respectivo diccionario; el análisis univariado consistió en la obtención de frecuencias absolutas y relativas de las variables cualitativas, y el análisis bivariado se llevó a cabo mediante la prueba de Chi cuadrado, previa comprobación de distribución mediante la Prueba Kolmogorov Smirnov; el estudio tuvo un nivel de confianza de $95 \%$ y un $p<0,05$.

\section{Resultados}

La muestra estuvo distribuida de manera equilibrada entre los pacientes de las instituciones públicas y privadas, la prevalencia resultó ser altamente significativa (tabla 1).

Tabla 1. Prevalencia de automedicación en pacientes de los centros de especialidades odontológicas y Centro De Salud Número 1 Azogues, 2017.

\begin{tabular}{clc}
\hline & $\mathrm{n}=201$ & \\
\hline Prevalencia & $\mathbf{N}^{\circ}$ & $\%$ \\
\hline $\mathrm{Si}$ & & 75 \\
\hline
\end{tabular}




\begin{tabular}{cll}
\hline & $\mathrm{n}=201$ & \\
\hline Prevalencia & $\mathbf{N}^{\circ}$ & $\%$ \\
\hline No & 52 & 25 \\
\hline & & \\
\hline Total & 201 & 100 \\
\hline
\end{tabular}

No. Valor absoluto

$\%$ porcentaje

Fuente: elaboración propia

En la entidad privada la automedicación fue más prevalente en sexo masculino y en la entidad pública en sexo femenino; sin diferencia estadísticamente significativa, $p=$ 0,67. De igual forma al relacionar edad y automedicación se demostró que en ambos grupos prevaleció la condición de automedicación de forma importante en relación a la no automedicación. (Tablas 2 y 3)

Tabla 2. Prevalencia de automedicación en pacientes de los centros de especialidades odontológicas y Centro De Salud Número 1 Azogues, 2017 de acuerdo con sexo.

\begin{tabular}{|c|c|c|c|c|c|c|c|c|}
\hline \multirow{5}{*}{ INSTITUCIÒN } & \multicolumn{7}{|c|}{ Automedicación } & \\
\hline & \multicolumn{3}{|c|}{ Se automedica } & \multicolumn{5}{|c|}{ No se automedica } \\
\hline & \multicolumn{3}{|c|}{ Sexo } & \multicolumn{5}{|c|}{ Sexo } \\
\hline & \multicolumn{2}{|l|}{ Masculino } & \multicolumn{2}{|l|}{ Femenino } & \multicolumn{2}{|l|}{ Masculino } & \multicolumn{2}{|c|}{ Femenino } \\
\hline & $n$ & $\%$ & $\mathrm{n}$ & $\%$ & $n$ & $\%$ & $n$ & $\%$ \\
\hline $\begin{array}{l}\text { Clínicas docentes } \\
\text { odontológicas }\end{array}$ & 24 & 60 & 56 & 51 & 12 & 57 & 19 & 61 \\
\hline $\begin{array}{c}{ }^{*} \text { Centro de Salud Número } \\
1 \text { Azogues }\end{array}$ & 16 & 40 & 53 & 49 & 9 & 43 & 12 & 39 \\
\hline Total & 40 & 100 & 109 & 100 & 21 & 100 & 31 & 100 \\
\hline
\end{tabular}

CHI CUADRADO p = 0,679

Fuente: elaboración propia

Tabla 3. Prevalencia de automedicación en pacientes de los centros de especialidades odontológicas y Centro De Salud Número 1 Azogues, 2017 de acuerdo con edad.

\begin{tabular}{cccccc}
\hline & $17-25$ & \multicolumn{7}{c}{$26-60$} & & \\
\hline & $\mathrm{n}$ & $\%$ & $\mathrm{n}$ & $\%$ & Total \\
\hline Si automedica & 56 & 76,72 & 93 & 72,66 & 149 \\
\hline No automedica & 17 & 23,28 & 35 & 27,34 & 52 \\
\hline Total & 73 & 100 & 128 & 100 & 201 \\
\hline
\end{tabular}

CHI CUADRADO $=0,52$

Fuente: elaboración propia 
Tanto en el sexo masculino como en el femenino los AINES constituyeron los fármacos más prevalentes en automedicación seguido de los analgésicos, sin encontrarse diferencia estadísticamente significativa, $p=0.165$ en el mismo sentido analizando los resultados del tipo de fármaco más auto suministrado según grupo etario lo constituyó el de 17 a 25 años, con AINES, seguido de analgésicos (tablas 4 y 5).

Tabla 4. Automedicación según el tipo de medicamento en centros de especialidades odontológicas y Centro De Salud Número 1 Azogues, 2017 según sexo.

\begin{tabular}{|c|c|c|c|c|c|c|}
\hline \multirow[b]{3}{*}{ FÁRMACO } & \multicolumn{4}{|c|}{ Sexo } & \multirow{2}{*}{\multicolumn{2}{|c|}{ Total }} \\
\hline & \multicolumn{2}{|c|}{ Masculino } & \multicolumn{2}{|c|}{ Femenino } & & \\
\hline & $\mathrm{n}$ & $\%$ & $\mathrm{n}$ & $\%$ & $\mathrm{n}$ & $\%$ \\
\hline No se Automedica & 21 & 34 & 31 & 22 & 52 & 26 \\
\hline AINES & 13 & 21 & 47 & 34 & 60 & 30 \\
\hline \multirow[t]{3}{*}{ Analgésicos } & 9 & 15 & 30 & 21 & 39 & 19 \\
\hline & \multicolumn{4}{|c|}{ Sexo } & \multirow{2}{*}{\multicolumn{2}{|c|}{ Total }} \\
\hline & \multicolumn{2}{|c|}{ Masculino } & \multicolumn{2}{|c|}{ Femenino } & & \\
\hline FÁRMACO & $\mathrm{n}$ & $\%$ & $\mathrm{n}$ & $\%$ & $\mathrm{n}$ & $\%$ \\
\hline Antibióticos & 6 & 10 & 6 & 4 & 12 & 6 \\
\hline Combinación de medicamentos & 8 & 13 & 18 & 13 & 26 & 13 \\
\hline No recuerda & 4 & 7 & 8 & 6 & 12 & 6 \\
\hline Total & 61 & 100 & 140 & 100 & 201 & 100 \\
\hline
\end{tabular}

CHI CUADRADO $p=0.165$

Fuente: elaboración propia

Tabla 5. Automedicación según el tipo de medicamento en centros de especialidades odontológicas y Centro De Salud Número 1 Azogues, 2017 según edad.

\begin{tabular}{|c|c|c|c|c|c|c|}
\hline \multirow[b]{3}{*}{ FARMACO } & \multicolumn{4}{|c|}{ Edad } & \multirow{2}{*}{\multicolumn{2}{|c|}{ Total }} \\
\hline & \multicolumn{2}{|c|}{17 a 25 años } & \multicolumn{2}{|c|}{26 a 60 años } & & \\
\hline & $n$ & $\%$ & $\mathrm{n}$ & $\%$ & $\mathrm{~N}$ & $\%$ \\
\hline No se Automedica & 17 & 23 & 35 & 27 & 52 & 26 \\
\hline AINES & 24 & 33 & 36 & 28 & 60 & 30 \\
\hline Analgésicos & 15 & 21 & 24 & 19 & 39 & 19 \\
\hline Antibióticos & 3 & 4 & 9 & 7 & 12 & 6 \\
\hline $\begin{array}{l}\text { Combinación de medica- } \\
\text { mentos }\end{array}$ & 10 & 14 & 16 & 13 & 26 & 13 \\
\hline No recuerda & 4 & 5 & 8 & 6 & 12 & 6 \\
\hline Total & 73 & 100 & 128 & 100 & 201 & 100 \\
\hline
\end{tabular}

CHI CUADRADO $p=0,915$

Fuente: elaboración propia 
En cuanto a la forma de presentación en ambos grupos de edad la más utilizada fue la de tabletas, cápsulas o comprimidos orales en los grupos de 17 a 25 y 26 a 60, superando notoriamente a las otras formas de presentación; lo mismo acontece en relación a sexo (tablas 6 y 7 ).

Tabla 6. Automedicación según presentación en los centros de especialidades odontológicas y Centro De Salud Número 1, Azogues, 2017, de acuerdo con edad.

\begin{tabular}{|c|c|c|c|c|c|c|}
\hline \multirow{3}{*}{$\begin{array}{c}\text { Presentación más utilizada en la } \\
\text { automedicación }\end{array}$} & \multicolumn{4}{|c|}{ Edad } & \multirow{2}{*}{\multicolumn{2}{|c|}{ Total }} \\
\hline & \multicolumn{2}{|c|}{17 a 25 años } & \multicolumn{2}{|c|}{26 a 60 años } & & \\
\hline & $\mathrm{n}$ & $\%$ & $\mathrm{n}$ & $\%$ & $\mathrm{n}$ & $\%$ \\
\hline No se automedica & 17 & 23 & 35 & 27 & 52 & 26 \\
\hline Tabletas, cápsulas o comprimidos orales & 51 & 70 & 83 & 65 & 134 & 67 \\
\hline Inyectables & 1 & 1 & 3 & 2 & 4 & 2 \\
\hline \multirow{3}{*}{$\begin{array}{c}\text { Presentación más utilizada en la } \\
\text { automedicación }\end{array}$} & \multicolumn{4}{|c|}{ Edad } & \multirow{2}{*}{\multicolumn{2}{|c|}{ Total }} \\
\hline & \multicolumn{2}{|c|}{17 a 25 años } & \multicolumn{2}{|c|}{26 a 60 años } & & \\
\hline & $n$ & $\%$ & $n$ & $\%$ & $\mathrm{n}$ & $\%$ \\
\hline Jarabes & 1 & 1 & 2 & 2 & 3 & 1 \\
\hline Combinaciones & 3 & 4 & 5 & 4 & 8 & 4 \\
\hline Total & 73 & 100 & 128 & 100 & 201 & 100 \\
\hline
\end{tabular}

Chi cuadrado $p=0,950$

Fuente: elaboración propia

Tabla 7. Automedicación según presentación en los centros de especialidades odontológicas y Centro De Salud Número 1 Azogues, 2017 de acuerdo con sexo.

\begin{tabular}{|c|c|c|c|c|c|c|}
\hline & \multicolumn{4}{|c|}{ Sexo } & \multirow{2}{*}{\multicolumn{2}{|c|}{ Total }} \\
\hline & \multicolumn{2}{|c|}{ Masculino } & \multicolumn{2}{|c|}{ Femenino } & & \\
\hline $\begin{array}{l}\text { Presentación más utilizada en la } \\
\text { Automedicación }\end{array}$ & $n$ & $\%$ & $\mathrm{n}$ & $\%$ & $\mathrm{n}$ & $\%$ \\
\hline No se Automedica & 21 & 34 & 31 & 22 & 52 & 26 \\
\hline Tabletas, cápsulas o comprimidos orales & 35 & 57 & 99 & 71 & 134 & 67 \\
\hline Inyectables & 2 & 3 & 2 & 1 & 4 & 2 \\
\hline Jarabes & 2 & 3 & 1 & 1 & 3 & 1 \\
\hline Combinaciones & 1 & 2 & 7 & 5 & 8 & 4 \\
\hline Total & 61 & 100 & 140 & 100 & 201 & 100 \\
\hline
\end{tabular}

CHI CUADRADO $p=0,115$

Fuente: elaboración propia 
En referencia al motivo de automedicación o patología asociada a ella, considerando edad y sexo se comprobó que el dolor dental prevaleció seguido muy distante del dolor de la muela del juicio y otras razones (tablas 8 y 9 ).

Tabla 8. Automedicación según patología asociada en los centros de especialidades odontológicas y Centro De Salud Número 1 Azogues, 2017, de acuerdo con sexo.

\begin{tabular}{|c|c|c|c|c|c|c|}
\hline \multirow{3}{*}{ Motivo de Automedicación } & \multicolumn{4}{|c|}{ Sexo } & & \\
\hline & \multicolumn{2}{|c|}{ Masculino } & \multicolumn{2}{|c|}{ Femenino } & \multicolumn{2}{|c|}{ Total } \\
\hline & $\mathrm{n}$ & $\%$ & $n$ & $\%$ & $n$ & $\%$ \\
\hline No se Automedica & 21 & 34 & 31 & 22 & 52 & 26 \\
\hline Dolor dental & 21 & 34 & 60 & 43 & 81 & 40 \\
\hline Dolor de encías & 2 & 3 & 4 & 3 & 6 & 3 \\
\hline Dolor de la muela del juicio & 6 & 10 & 22 & 16 & 28 & 14 \\
\hline \multirow[t]{2}{*}{ Hinchazón de la cara } & 4 & 7 & 7 & 5 & 11 & 5 \\
\hline & \multicolumn{4}{|c|}{ Sexo } & & \\
\hline \multirow{2}{*}{ Motivo de Automedicación } & \multicolumn{2}{|c|}{ Masculino } & \multicolumn{2}{|c|}{ Femenino } & \multicolumn{2}{|c|}{ Total } \\
\hline & $\mathrm{n}$ & $\%$ & $n$ & $\%$ & $n$ & $\%$ \\
\hline Otros & 7 & 11 & 16 & 11 & 23 & 11 \\
\hline Total & 61 & 100 & 140 & 100 & 201 & 100 \\
\hline
\end{tabular}

Chi cuadrado $p=0,481$

Fuente: elaboración propia

Tabla 9. Automedicación según patología asociada en los centros de especialidades odontológicas y Centro De Salud Número 1 Azogues, 2017 de acuerdo con edad.

\begin{tabular}{|c|c|c|c|c|c|c|}
\hline \multirow{3}{*}{ Motivo de automedicación } & \multicolumn{4}{|c|}{ Edad } & \multirow{2}{*}{\multicolumn{2}{|c|}{ Total }} \\
\hline & \multicolumn{2}{|c|}{17 a 25 años } & \multicolumn{2}{|c|}{26 a 60 años } & & \\
\hline & $n$ & $\%$ & $n$ & $\%$ & $\mathrm{n}$ & $\%$ \\
\hline No se Automedica & 17 & 23 & 35 & 27 & 52 & 26 \\
\hline Dolor dental & 30 & 41 & 51 & 40 & 81 & 40 \\
\hline Dolor de encías & 4 & 5 & 2 & 2 & 6 & 3 \\
\hline Dolor de la muela del juicio & 9 & 12 & 19 & 15 & 28 & 14 \\
\hline Hinchazón de la cara & 4 & 5 & 7 & 5 & 11 & 5 \\
\hline Otros & 9 & 12 & 14 & 11 & 23 & 11 \\
\hline Total & 73 & 100 & 128 & 100 & 201 & 100 \\
\hline
\end{tabular}

Chi Cuadrado $p=0.701$

Fuente: elaboración propia 
Respecto a los factores que influyen en la automedicación odontológica de los pacientes estudiados, se encontró que en su mayoría son los familiares o el farmacéutico quienes intervienen en la decisión de automedicarse. (Tabla 10)

Tabla 10. Automedicación según recomendación en centros de especialidades odontológicas y Centro De Salud Número 1 Azogues, 2017

\begin{tabular}{lll}
\hline \multicolumn{1}{c}{ Se automedica por recomendación de } & Frecuencia & Porcentaje \% \\
\hline No se automedica & 52 & 25,9 \\
\hline Familiares & 65 & 32,3 \\
\hline Amistades & 9 & 4,5 \\
\hline Farmacéuticos & 66 & 32,8 \\
\hline Medios de comunicación & 9 & 4,5 \\
\hline Total & 201 & 100,0 \\
\hline
\end{tabular}

Fuente: elaboración propia

Se contrastó automedicación en relación al tipo de entidad sanitaria y ocurrió que en mayor proporción se automedican los pacientes que acuden a clínicas privadas en relación a las públicas, sin que haya relación estadísticamente significativa. $p=0,460$ (tabla 11).

Tabla 11. Comparación de automedicación según el tipo de institución.

\begin{tabular}{|c|c|c|c|c|c|c|}
\hline \multirow[b]{3}{*}{ Institución } & \multicolumn{4}{|c|}{ Automedicación } & \multirow{2}{*}{\multicolumn{2}{|c|}{ Total }} \\
\hline & \multicolumn{2}{|c|}{$\mathrm{Si}$} & \multicolumn{2}{|c|}{ No } & & \\
\hline & $n$ & $\%$ & $n$ & $\%$ & $n$ & $\%$ \\
\hline $\begin{array}{l}\text { Clínicas docentes } \\
\text { odontológicas }\end{array}$ & 80 & 54 & 31 & 60 & 111 & 55 \\
\hline \multicolumn{7}{|l|}{ Centro de Salud } \\
\hline Número 1 & 69 & 46 & 21 & 40 & 90 & 45 \\
\hline \multicolumn{7}{|l|}{ Azogues } \\
\hline Total & 149 & 100 & 52 & 100 & 201 & 100 \\
\hline
\end{tabular}

CHI CUADRADO $p=0,460$

Fuente: elaboración propia 


\section{Discusión}

En el Ecuador existen pocos estudios para determinar la prevalencia sobre automedicación en general y menos aún en odontología. Los datos recabados como referentes nacionales se han extraído más de notas periodísticas, que de estudios científicos, con la rigurosidad que ello necesita; por lo que se propuso en este estudio investigar la automedicación en el campo de la odontología en las provincias de Azuay y Cañar a través de centros de referencia de salud, uno público y los otros privados. En este sentido se tomaron en cuenta las ciudades mencionadas, mismas que están ubicadas en el sur del Ecuador, muy cercanas una de la otra, a tan solo $30 \mathrm{~km}$ de distancia, por tanto, comparten características geográficas, climáticas y poblacionales muy similares. Ello facilitó la realización del presente trabajo investigativo; desarrollado en una entidad pública Centro de Salud Número 1 de Azogues y en una entidad privada Clínicas de Especialidades Odontológicas de la Universidad Católica de Cuenca, donde se tomaron datos de los pacientes que acudieron a consulta externa odontológica.

El estudio se realizó con 201 pacientes de ambos sexos y en un rango de edad entre 17 a 60 años, de los cuales 111 pertenecían a la entidad privada y 90 a la entidad pública, las variables principales que se examinaron fueron: sexo, edad, tipo de medicamento más usado, patología relacionada, persona que recomienda, edad de primera automedicación y comparación entre las dos instituciones mencionadas anteriormente; indicadores utilizados también en los estudios de Bisinoto (3), Conhi (1), Pumahuanca (2).

En referencia a sexo, el presente estudio determinó que, en institución pública, la automedicación fue discretamente mayor (9\%más) en sexo femenino, en tanto que en las privadas la misma diferencia porcentual se observó pero en relación de mayor prevalencia para el sexo masculino, sin encontrarse diferencia estadísticamente significativa, este aspecto coincide con los estudios de Bisinoto (3), Pumahuanca (2), Guillem (12), Castillo (9), Dresh (13).

Al considerar la edad en este estudio se pudo evidenciar que en ambos grupos prevaleció la condición de automedicación de forma importante en relación a la no automedicación 76,72 y 72,65 \%, resultando quizá, por los resultados mayor la necesidad de automedicarse en los más jóvenes y aun cuando no existen muchos estudios que analicen esa relación lo detectado coincide con lo reportado por Bisinoto et al. (3), Pumahuanca (2), Dresh (13) y difiere con el estudio de Zapata (14).

Partiendo de la premisa que todo medicamento tiene implícita una acción terapéutica y preventiva, ello no quiere decir que potencialmente estarían siendo 
elementos de riesgo, con efectos colaterales y adversos; por ello es que en los estudios de utilización de medicamentos (EUM) se analizan los parámetros de uso, en qué casos se los debe usar, cuáles serían esas indicaciones, para sortear efectos desfavorables derivados de una administración equivocada, y concomitantemente una autoadministración de manera irresponsable, especialmente con los antibióticos y los AINES (4). En el presente estudio los fármacos asociados a la automedicación con mayor prevalencia fueron los de la familia de AINES, sin que exista relación significativa entre esas variables coincidiendo con los estudios de Kumar (15), Azevedo (16); el estudio de Conhi (1) denota a diferencia del presente la preferencia por los analgésicos; de igual forma los estudios de Guillem (12), Carneiro (17), Anyanechi (5), Arun (7),revelaron la preferencia de los analgésicos para automedicarse.

Relacionando edad y tipo de fármaco prevaleció la automedicación dental de AINES sobre los analgésicos, es decir, el grupo etario más joven prefiere los AINES resultando coincidente con varios estudios en donde los AINES son los más requeridos para automedicación $(1,2,12,17)$.

La forma farmacéutica más usada por los pacientes para la automedicación odontológica fueron las tabletas, cápsulas o comprimidos orales; esto tanto para el sexo femenino como masculino con un 57 y 71 \% respectivamente. De igual manera, al relacionar con la edad coincide que la forma farmacéutica más usada son las tabletas, sin encontrar relación significativa; de todos modos, independientemente de sexo y edad algunos estudios reportan que esta presentación oral suele ser la más solicitada con fines de automedicación (1), (12,17).

El motivo de automedicación dental es un aspecto de suma importancia en el paciente odontológico, específicamente hablando del dolor dental, en el presente estudio este aspecto representó el factor número uno que obligó al paciente a automedicarse dejando muy por debajo a otras condiciones como el dolor de la muela del juicio o la hinchazón de la cara. Esta situación ocurrió tanto al considerar sexo como edad; la misma tendencia se encuentra en varios estudios reportados en Brasil, Perú, India y Nigeria e inclusive coincidiendo que la molestia del dolor dental afecta a pacientes que acuden a instituciones públicas y privadas $(1,3,5,7,13,17)$.

Con respecto a quien recomienda la automedicación odontológica en este estudio se encontró al farmacéutico y familiares con un 32,8 \% y 32,3 \% respectivamente, como los principales responsables de generar este hábito; siendo estos datos iguales a muchos encontrados en varios países del mundo, se podría inferir que el farmacéutico sea un profesional de la rama o un empírico responsable de la venta de fármacos, se constituye como uno de los principales referentes para la automedicación (1,2,7,12-14,18,19). 
Como un aspecto complementario el presente trabajo indagó sobre la fecha de primera automedicación, para ello y tratando en lo posible de controlar un sesgo de memoria, se agruparon por grupos etarios dando una adecuada opción de que el paciente encuestado escoja el grupo dentro del cual ubique su primera automedicación; obteniendo como resultado que el $72 \%$ lo hicieron a edades tempranas entre los 17 a 25 años; esto coinciden con los resultados obtenidos en países como Brasil y Perú $(2,3,8)$.

La responsabilidad sobre los riesgos de automedicación odontológica de los participantes de este estudio reveló que el 65,2 \% tiene conocimiento de que este hábito causa múltiples daños a su salud, de igual manera varios estudios afirman que automedicarse en general constituye actualmente una problemática de salud pública que desencadena importantes deterioros en la comunidad, ya que algunas acciones favorecen a su práctica, pudiendo citar las que con mayor frecuencia impulsan a que la población practique este hábito: aumento de propaganda de medicamentos en los medios de comunicación, falta de acceso a información sobre este tema y sobre todo el desarrollo de la industria farmacéutica que despliega una disponibilidad cada vez mayor de medicamentos de libre acceso (18-20). En países como la India y Perú se ha demostrado que el desconocimiento sobre este tema es muy grande y que conlleva a una automedicación irresponsable lo que produce falta de conciencia sobre los daños que ocasiona esta práctica errónea en la salud particularmente la odontológica $(2,7)$.

La OMS se pronuncia sobre la gran prevalencia de automedicación incluida la dental, atribuyendo que dichas prácticas son propias de países en vías de desarrollo, debiendo reconocer por tanto que países como Ecuador estarían en ese contexto. En coherencia a esta afirmación, el presente estudio tuvo como resultado una automedicación odontológica del 75 \%, lo que quiere decir que, de los 201 pacientes encuestados, 149 optaron por la práctica de este hábito al referir cierta dolencia de origen dental; esto concuerda con los estudios de Kumar (15), Jain (18), Pumahuanco (2), Anyanechi Nigeria (5) en donde la prevalencia de automedicación fue similar. Sin embargo, en países europeos y otros de Sudamérica como Brasil cuyo desarrollo ha demostrado sostenibilidad también afirman prevalencias altas de automedicación constituyéndose en definitiva en un fenómeno global y universal $(3,9,21,22)$.

En la comparación entre entidad pública (Centro de Salud Número 1 Azogues) y privada (Clínicas de especialidades odontológicas) en ambas instituciones prevaleció la práctica de la automedicación con un ligero predominio de la privada en relación a la pública, aspecto que se presta para el análisis en razón de que las personas que acuden a centros privados traen implícita una educación formal lo que llevaría a la conciencia de no automedicarse; ello no aconteció en este estudio, por tanto es necesario realizar futuras investigaciones tomando en consideración elementos 
como variables sociodemográficas, nivel de instrucción, aspectos de orden cultural, entre otras, que permitan concluir cuáles son los factores determinantes para la automedicación y particularmente la odontológica.

\section{Conclusiones}

La prevalencia de automedicación odontológica la tuvieron casi la totalidad de la muestra estudiada, con diferencias discretas respecto al sexo, y a los grupos etarios, evidenciándose quizá en el grupo más joven una mayor condición de automedicación. El riesgo está latente al evidenciarse la autoadministración bajo recomendación en su mayoría por parte de farmacéuticos de AINES cuyos riesgos y efectos por uso indiscriminado, son potencialmente graves. Si bien el estudio evidenció que muchos lo hacían en razón de la emergencia del dolor dental, no es menos cierto que toman la vía más fácil cuando lo adecuado es acudir a consulta para posterior a un diagnóstico adecuado sea el profesional quien prescriba la medicación correcta; en razón de esto los estudios de automedicación son cada vez más necesarios para contribuir a mejorar la conciencia sanitaria de un uso totalmente racional de los medicamentos.

\section{Referencias}

1. Conhi A, Castillo-Andamayo DE, Castillo-López CD. Automedicación odontológica de pacientes que acuden a una institución pública y privada, Lima-Perú. Rev Estomatol Herediana. 2015; 25(3): 205-10. doi: https://doi.org/10.20453/reh.v25i3.2623

2. Pumahuanca O, Cruz T. Automedicación con Aines por Pacientes con Odontalgia en la Consulta Pública y Privada. Rev Evid Odontol.Clinic. 2016; 2(2): 30-33.

3. Bisinoto M, Parreiras, MA, Nogueira Guimarães MH, Silva de Castilho L. Fatores associados à automedicação em um serviço brasileiro de emergência odontológica. Rev Pesq Bras Odontoped Clin Integr João Pessoa. 2012; 12(1): 65-69.

4. Karaben VE, Rea AE, Ramirez LI. Prescripciones de antibióticos para patologías bucodentales. Rev Nac Odontol. 2017; 13(26). doi: https://doi.org/10.16925/od.v13i25.1883

5. Anyanechi S. Toothache and self-medication practices: A study of patients attending a Niger Delta Tertiary Hospital in Nigeria. Ann Med Health Sci Res. 2014; 4(6): 884-888. doi: 10.4103/2141-9248.144896. 
6. Adedapo H, Lawal A.O, Adisa AO, Adeyemi BF. Non doctor consultations and self medication practices in patients seen at a tertiary dental center in Ibadan. Indian. J Dent Res. 2012;22:795-8.

7. Simon AK, Rao A, Rajesh G, Shenoy R, Pai MB. Trends in self medication for dental conditions among patients attending oral health outreach programs in coastal Karnataka, India. J Pharmacol. 2015; 47(5): 524-529. doi: 10.4103/0253-7613.165195

8. Nogueira-Castillo M, Orejuela-Ramírez F, Andamayo-Flores D, Castillo-Andamayo D. Factores asociados a la automedicación en pacientes que acuden a Servicios de Odontología de Hospitales del Perú. Rev Estomatol Herediana. 2018; 28(2). doi: https://doi.org/10.20453/reh. v28i2.3322

9. Castillo N. Frecuencia y factores asociados a la automedicación en pacientes que acuden a servicios de odontología de instituciones públicas de Piura, Cajamarca e Iquitos [Tesis doctoral]. Perú: Universidad Peruana Cayetano Heredia, Facultad de Estomatología; 2015.

10. Escalona CD, Scarpeccio R, Quiñònez M. Automedicación analgésica en pacientes que acuden al Servicio de Odontología del Hospital General Dr. Luis Razetti. Barinas estado Barinas, Venezuela. Rev Inst Nac Hig Rafael Rangel. 2017; 48(1-2): 9 - 26

11. El telégrafo. wwweltelegrafocomec [Internet]. [Consultado 26 julio 2014]. Disponible en: http://www.eltelegrafo.com.ec/noticias/sociedad/4/mas-del-50-de-farmacos-son prescritos-sin-una-receta-documento

12. Guillem P, Francès-Bozal F, Gimenez-Fernández F, Sáiz-Sánchez C. Estudio sobre Automedicación en Población Universitaria Española. Rev Clin Med Fam. 2010; 3(2): 99-103.

13. DreshA.Conhecimento dos pacientessobremedicamentos prescritos porodontólogos nosuldo Brasil. Ciência \& Saúde Coletiva, 2016;21(2): 475-483. doi: 10.1590/1413-81232015212.17732014

14. Zapata A. Nivel Socioeconómico y la Automedicación dental de los pacientes que acuden a la Clínica Dental Víctor Raúl Haya de la Torre en el Distrito de Breña. [Tesis doctoral]. Perú: Universidad Inca Garcilaso de la Vega; 2017.

15. Kumar R, Goyal A, Padhy BM, Gupta YK. Self-medication practiceand factors influencing it among medical and paramedical students in India: A two- period comparative crosssectional study. J Nat Sci Biol Med. 2016; 7(2): 143-148. doi: 10.4103/0976-9668.184700

16. Azevedo FHC, Fontenele JCB, Miranda GL. Factores asociados con pacientes tratados automedicación en una oficina dental, en la ciudad de Piracuruca. Rev Interd. 2014; (3): 83-90. 
17. Carneiro DS, Valoz FEF, Silva FACD, Pereira CM. Automedicação entre acadêmicos de odontologia da Universidade Paulista Goiânia-Goiás. RFO UPF. 2011; 16(3).

18. Jain A, Bhaskar DJ, Gupta D, Agali C, Yadav P,Khurana R. Practice of Self-Medication for Dental Problems in Uttar Pradesh, India. Oral Health Prev Dent. 2016; 14(1):5-11. doi: 10.3290/j.ohpd.a35000.

19. Aguedo A. Relación entre la automedicación para el manejo del dolor dentario y el nivel socioeconómico-cultural de los pobladores del distrito de Independencia. Huaraz, 2007. Rev Odontología Sanmarquina. 2008; 11(2): 78-82.

20. de Pablo MM. La automedicación en la cultura Universitaria. Rev de Investigación. 2011; 35(73): 219-239.

21. Silva LSF, Costa AMD, Terra FDS, Zanetti HHV, Costa RD, Costa MD. Automedicação em acadêmicos de cursos de graduação da área da saúde de uma universidade privada do Sul do estado de Minas Gerais. Clín Cient. 2011; 10(1): 57-63.doi: http://dx.doi.org/10.17058/reci.v2i4.3324

22. WHO. Guidelines for the medical assessment of drugs for use in self-medication. Copenhague: WHO-Regional Office for Europe. 1986. doi: https://doi.org/10.1016/S1138-3593(08)71865-3Get r 